\title{
Studies on the Relationships between Clean/fossil Energy Consumption and Sustainable Development in China
}

\author{
Kezhou LI, Lai Leng LAO, Qiang YI, Hsing Hung CHEN \\ School of Business, Macau University of Science and Technology, Macau, China
}

\begin{abstract}
: this paper expects to examine the causal relationship of clean/fossil energy consumption with economic growth, environmental issues, and social problems. Especially, China consumed 2,735 million tons of oil equivalent of primary energy including $86 \%$ of fossil energy and $12.3 \%$ of clean energy, accounting for $21.9 \%$ of the world's total in 2013, and the consumption in China over passed that in the USA since 2010. Then, the importance of examining the relationships between clean/fossil energy consumption and sustainable development is obvious and urgent. Specifically, this study examines the causal relationship within a multivariate panel co-integration/error correction framework, which combines the cross-section and time series data while allowing for heterogeneity across different provinces.
\end{abstract}

KEYWORD: Clean and fossil energy consumption; sustainable development; ecological environment; social development; economic growth

\section{INTRODUCTION}

In light of the recent concerns over greenhouse gas (GHG) emissions produced from fossil fuel-fired energy sources, the high volatility of oil and gas prices in international markets, and the dismatched geopolitical landscape forcing the country to highly depend on foreign energy sources, a stable supply of alternative energy is a timely issue in China. In order to achieve a sustainable balance between economic growth and environmental protection, the governments have promoted policies such as renewable energy production tax credits, installation rebates for renewable energy systems, renewable energy portfolio standards, and the establishment of markets for renewable energy certificates (Zhang et al., 2011; Hu and $\mathrm{Xu}, 2013)$. In China, the share of natural gas in the total energy consumption had grown from $1.8 \%$ in 1995 to $3.5 \%$ in 2007 , and the ratio is likely to reach $10 \%$ by 2020 , with energy consumption equaling to 200 billion cubic meters (Bcm) (Cui, 2008). Because the demand for domestic natural gas use has grown more quickly than the supply in China, the National Development and Reform Commission (NDRC) forecasted that the gap of natural gas demand and supply would reach $50 \mathrm{Bcm}$ in 2015 and $80 \mathrm{Bcm}$ in 2020 (Shi et al., 2010). In addition, rapid growth in electricity demand and suitable adjustment in power structure have accelerated construction of nuclear power plants in China. The installed nuclear power capacity in 2012 was $18 \mathrm{GW}$. However, the total installed nuclear power capacity will be over $70 \mathrm{GW}$ by 2020 , $75 \%$ higher than government target set in the Medium- and Long-term Nuclear Power Development Plan (Wang, 2009). According to national hydropower resources survey, the theoretical reserve capacity of hydropower in China is $694 \mathrm{GW}$, technical exploitable capacity $542 \mathrm{GW}$, and economic exploitable capacity $402 \mathrm{GW}$ ( $\mathrm{Li}$ and Shi, 2006). Hydropower is the largest source of renewable energy in China, making up $82 \%$ of China's renewable portfolio in 2011. With an installed capacity of $231 \mathrm{GW}$ in 2011 , hydro power accounted for roughly $7.8 \%$ of the national total installed capacity (Cohen and Siu, 2012). The capacity is planned to reach $300 \mathrm{GW}$ by 2015 (Cohen and Siu, 2012). Finally, the installed fossil energy capacity in China was 2,357 million tons of oil equivalent, or $86 \%$ of the total installed capacity in 2013 (BP, 2014). Coal accounted for $68.5 \%$ of all power generated in 2102, far above the world average of $40 \%$ (IEA, 2007). If China maintains the rate of coal-fired power, 2.0 billion tons of power coal will be required by 2020 (NDRC, 2007). Actually, China uses more coal than the combination of the United States, the European Union and Japan (BP, 2008). Thus, the study to examine the 
relationship between clean/fossil energy consumption and economic development is urgent and significant.

Numerous publications have adopted practical examples to examine the relationship of economic growth with energy consumption/production and other parameters (Apergis and Payne, 2010; Menegaki, 2011; Silalertruksa et al., 2012; Bildirici, 2013). After reviewing extensive literatures, Das et al. (2013) summarize that the mechanism of how renewable energy corresponds to economic development, reduces carbon dioxide emissions, substitutes exhausted fossil fuel and balances regional development was still uncertain (such as growth, conservation, feedback and neutrality hypothesis). Especially, both clean energy including natural gas, hydropower, and nuclear energy, as well as fossil energy including petroleum and coal, have only been simultaneously included in limited studies, and these papers have adopted methods such as Granger causality, panel co-integration test, and panel error correction model (Apergis and Payne, 2010; Payne, 2011; Kum et al., 2012; Das et al., 2013). However, this study examines the causal relationship within a multivariate panel cointegration/error correction framework. The framework combines the cross-section and time series data and allows for heterogeneity across different provinces in China. In addition, the exclusion of relevant variables makes the forecasts inconsistent and biased, and no-causality in a bivariate system can result from neglected variables (Lütkepohl, 1982). Then, this research includes carbon dioxide emissions, income per capita, fixed capital formation and labor force as additional variables because clean/fossil energy consumption alone might not be strong enough to spur economic growth.

\section{METHOD}

\subsection{Proposed hypotheses}

In order to examine the causal relationship of clean/fossil energy consumption with economic growth, environmental issues and social problems, the following hypotheses are made:

\section{Hypothesis (a): The causal relationship of natural logarithm of real GDP per capita with change of GHG emissions should be negative in a specific period.}

Menegaki (2011) used annual panel data ranging from 1997 to 2007 for 27 European countries to examine the causal relationship of real GDP per capita with greenhouse emissions equivalents with base year 1990, and concluded that their relationship was apparently opposite.
Hypothesis (b): The causal relationship of natural logarithm of real GDP per capita with change of clean energy consumption should be positive in a specific period.

Many studies have shown that a substantial increase in energy consumption, especially in renewable energy consumption, has a significant relationship with economic development (Apergis and Payne, 2010). In addition, Economides and Wood (2009) stated that natural gas was clearly destined to help achieve two important energy goals for the $21^{\text {st }}$ century: providing the sustainable energy supplies and promoting economic development. On the other hand, Das et al. (2013) summarized that the causal relationship of real GDP per capita with the clean energy consumption was uncertain in different countries.

\section{Hypothesis (c): The causal relationship of natural logarithm of real GDP per capita with change of fossil fuel-based energy consumption should be positive in a specific period.}

Tsani (2010) employed Toda and Yamamoto model to investigate the casual relationship between different levels of energy consumption and economic growth for Greece for the period 1960 through 2006. Empirical evidence suggests that there is a bidirectional causal relationship between industrial and residential energy consumption to real GDP but this is not the case for the transport energy consumption with causal relationship being identified in neither direction.

\section{Hypothesis (d): The causal relationship of natural logarithm of real GDP per capita with change of income per capita should be positive in a specific period.}

Weber (2012) used gas consumption and production data combined with economic data to estimate how a substantial increase in the value of gas consumption and production from 1999 to 2008 affected salary income in the country. The results indicate that a natural gas boom should increase salary income because of the combination of a greater demand for labor, an increase in the number of jobs, and a raise in the rent payments to private and public resource owners.

\section{Hypothesis (e): The causal relationship of natural logarithm of real GDP per capita with change of real gross fixed capital formation should be positive in a specific period.}

The existence of supporting schemes for renewable energy represents an opportunity to reduce the risk by ensuring a fixed income for a 
certain period of time, and reflects the need to ensure the interests from private investors for technological innovation (Carneiro and Ferreira, 2012). The supporting policies, such as regulated feed-in tariffs, installation rebates for renewable energy systems, investment grants, tradable green certificates, and tax incentives, may incur lots of investment capitals. Apergis and Payne (2010) used annual panel data from OECS to examine the causal relationship of the natural logarithm of renewable energy consumption (defined in millions of kilowatt hours as net geothermal, solar, wind, wood, and waste electric power consumption) with the natural logarithm of real gross fixed capital formation in constant 2005 US dollars and other parameters, and concluded that their relationship was significant.

\section{Hypothesis (f): The causal relationship of natural logarithm of real GDP per capita with change of labor force should be positive in a specific period.}

After using annual panel data ranging from 1998 to 2008 in nine developed countries to examine the causal relationship of the change of GDP per capita with the change of labor force and other parameters, Wolde-Rufael and Menyah (2011) concluded that the relationship was positive with significance.

\subsection{Research methodology}

In this study, panel data is applied due to three major reasons. First, each province in China has quite different cultural backgrounds and specific characteristics. The use of panel data can effectively control the heterogeneity of individual province. Second, since only 15 provinces/places have sufficient data, there are not enough observations for regression. The employment of panel data can solve the problem. Third, from the perspective of econometrical study, panel data can provide us more degrees of freedom, more information, more variation, and less collinearity (Wang, 2008). Accordingly, the paper will employ panel data models to simulate the multi-variable regressions.

To examine the presence of unit roots in a series, Levin-Lin-Chu (LLC) test, augmented Fisher Dickey-Fuller (Fisher-ADF) test and Fisher PhillipsPerron (Fisher-PP) test are well accepted methods. When co-integration test is applied to verify the stability of a system, Kao residual test is well accepted (Kao and Chiang, 2000). When employing the panel data model, three alternatives are pooled ordinary least square (OLS) regression model, fixed effect (FE) regression model, and random effect (RE) regression model. When likelihood test is passed, the FE regression model is more appropriate. When Hausman test is passed, the RE regression model is more suitable. Otherwise, the OLS regression model can be used. However, if both likelihood and Hausman tests are passed (in the paper, the number of provinces $(N)$ is greater than the number of year $(T)$, the FE model is more suitable if panel data is not randomly sampled (Hausman, 1978; Green, 2003). Oppositely, the RE model is more appropriate if panel data is randomly sampled.

Before simulating the regressions, several potential econometric problems need to be checked. The first one is collinearity between explanatory variables. People may have good reason to suspect a collinearity relation between variables, such as labor force and average income, because they may impact each other. This may be a critical problem if we employ just cross-sectional data. As mentioned above, panel data model can basically solve the collinearity problem. The second problem is heteroscedasticity among cross-sectional data. To solve this problem and possible cross-sectional correlation problem, we employ regressions that report Driscoll-Kraay standard errors. Such kinds of regressions are more robust if heteroscedasticity and cross-sectional correlation problems exist (Driscoll and Kraay, 1998). In addition, because the panel data in the case study has the characteristic of $\mathrm{N}>\mathrm{T}$, the problems of serial correlation and panel unit root may be reasonably ignored.

\section{RESULTS}

The panel data sets are examined by three regression models: ordinary least square (OLS) regression model, fixed effect (FE) regression model, and random effect (RE) regression model. Since Hausman tests are passed to support the original hypothesis ( $p$ value>0.05), FE regression models are not suitable to be employed. In addition, as mentioned previously, the RE model is more suitable if panel data is not randomly sampled and the number of observations for places is greater than the number of observations for years. Then, we have the panel data sets examined by RE regression model. Since the number of observations for places is not equal to the number of years, cross-section weights should be employed. Then, based on the unbalanced panel data sets consisting of 15 places in China over 2004-2013, RE regression model is adopted, and the results are obtained. In order to reduce a random residual $\left(\varepsilon_{i, t}\right)$ to reach an acceptable level, Generalized Least Square (GLS) estimation is employed to adjust failed homoscedastic hypothesis, mutual independence, and non-serial correlation. RE regression model is rerun over 2004-2013, and the results are shown in Table 1. 
Table 1 The final results for RE regression model

\begin{tabular}{|lll|}
\hline Variable & Coefficient & Prob. \\
\hline$\alpha$ & -11.98212 & 0.0000 \\
\hline Clean Energy & 0.420010 & 0.0000 \\
\hline Fossil-based Energy & 0.422037 & 0.0000 \\
\hline GHG Emissions & 0.581632 & 0.0073 \\
\hline Labor Force & -0.827230 & 0.5809 \\
\hline Fixed Capital & 0.400186 & 0.0915 \\
\hline \hline R-squared & 0.675557 & \\
\hline Adjusted R-squared & 0.661944 & \\
\hline S.E. of regression & 0.278975 & \\
\hline F-statistic & 8.144682 & \\
\hline Prob(F-statistic) & 0.000000 & \\
\hline
\end{tabular}

\section{CONCLUSION}

Past works, using hypotheses, including conversation, growth, feedback, and neutrality hypothesis, have tried to examine the causal relationship between real GDP per capita and some variables, such as energy consumption and income per capita, for a country during a specific period. Some models employed include the conventional Granger causality, panel co-integration test and panel error correction model, Toda-Yamamoto long-run causality, and bootstrap-corrected causality. However, to obtain an average solution for a country while considering the heterogeneity across different provinces, a multivariate panel co-integration/error correction framework combining the cross-section and time series data is more suitable. Especially, with the high degree of capital and labor mobility in China, the theory of optimum currency areas has been proved in China (Mundell, 2000). Then, employing panel data models to simulate the multivariable regressions to find an average correlation coefficient for a country among variables should be more meaningful.

Many countries are vigorously promoting lowcarbon economy with high efficiency and low emission, actively developing low carbon technology, and devising industrial, energy, technology, trade policies to develop green industries. Therefore, to promote a low-carbon economy and to compete in international green industries, China should utilize its characterized technologies and advantages effectively. On the demand side, the government should build a smart and convenient living environment with energy conservation and carbon reduction. These include intelligent and convenient transportation networks, green buildings, energy efficiency and green energy infrastructures, and low-carbon communities and cities. In addition, a complete market mechanism and legal system foundation for energy conservation and carbon reduction should be built, such as 'carbon dioxide trading law' and 'greenhouse gas reduction law', so that the external cost can be internalized and carbon reduction ability can be enhanced. On the supply side, the government should strive to promote the 'New Policy on Clean Energy Utilization' and 'Renewable Energy Promotion Law' projects, to achieve a total renewable energy device capacity accounted for more than $30 \%$ of total energy capacity in 2030 .

\section{REFERENCES}

[1] Marchand, J. 2010. Local Labor Market Impacts of Energy Boom-Bust-Boomin Western Canada, University of Alberta Working Paper No. 2010-2017.

[2] Mundell, R. 2000. Currency areas, volatility and intervention. Journal of Policy Modeling 22, 281-299.

[3] NDRC, 2007. Medium- and long-term nuclear power development plan during 2005 through 2010. Beijing, P.R. China: The National Development and Reform Commission; 2007.

[4] Omu, A., Choudhary, R., Boies, A. 2013. Distributed energy resource system optimization using mixed integer linear programming. Energy Policy 61, 249-266.

[5] Papyrakis, E., Gerlagh, R. 2007. Resource abundance and economic growth in the United States. European Economic Review 51, 1011-1039.

[6] Payne, J.E. 2011. US disaggregate fossil fuel consumption and real GDP: an empirical note, Energy Sources. Economics, Planning and Policy 6(1), 63-68.

[7] Paul, B.P., Uddin, G.S. 2011. Energy and output dynamics in Bangladesh, Energy Economics 33, 480-487.

[8] Reynolds DB, Kolodziej M. 2008. Former Soviet Union oil production and GDP decline: granger-causality and the multi-cycle Hubbert curve. Energy Economics 30, 271289.

[9] Shi, G.H., Jing, Y.Y., Wang, S.L., Zhang, X.T. 2010. Development status of liquefied natural gas industry in China. Energy Policy 38, 7457-7465. 